\title{
A Computational Technique for Determining the Class Number of a Pure Cubic Field
}

\author{
By Pierre Barrucand, H. C. Williams and L. Baniuk
}

\begin{abstract}
Two different computational techniques for determining the class number of a pure cubic field are discussed. These techniques were implemented on an IBM/370-158 computer, and the class number for each pure cubic field $Q\left(D^{1 / 3}\right)$ for $D=2,3, \ldots, 9999$ was obtained. Several tables are presented which summarize the results of these computations. Some theorems concerning the class group structure of pure cubic fields are also given. The paper closes with some conjectures which were inspired by the computer results.
\end{abstract}

1. Introduction. The theory of pure cubic fields $Q\left(D^{1 / 3}\right), D$ rational, was founded in 1892 by Markov [12]; in his paper he gives some class numbers and fundamental units, not always in an explicit form. In [7] Dedekind describes a method for determining the class number of a pure cubic field $Q\left(D^{1 / 3}\right)$. He also gives a short table of class numbers for some small values of $D$. Cohn [6] implemented Dedekind's method on a computer and obtained class numbers for some fields in which he could easily determine the regulator. Cohn's technique was modified somewhat by Beach, Williams, and Zarnke in [4] , and class numbers were obtained for $Q\left(D^{1 / 3}\right)$ for $D=2,3, \ldots$, 999. Other tables of class numbers have been calculated by hand by Cassels [5] and Selmer [14]. It should also be mentioned that Angell [1] has recently given a list of class numbers for all cubic fields with negative discriminant greater than $-20,000$.

The purpose of this paper is to present a new technique for determining the class number of $Q\left(D^{1 / 3}\right)$. This method is much faster than the computational technique of [6] and [4]. The algorithm was implemented on a computer and the class numbers for $Q\left(D^{1 / 3}\right)$ obtained for $D=2,3, \ldots, 9999$. The total number of these fields is 8122. We also describe here some of the results of these calculations.

2. Some Properties of Pure Cubic Fields. Let $K$ be any cubic field with discriminant $\Delta$. If $\zeta_{K}(s)$ is the Riemann zeta function in $K$, we have

$$
\Phi(s)=\zeta_{K}(s) / \zeta(s)=\sum_{j=1}^{\infty} \alpha(j) j^{-s} .
$$

Here $\alpha(j)=\Sigma_{d \mid j} \mu(d) F(j / d)$, where $F(n)$ is the number of distinct ideals of norm $n$ in $K$. Also, $C R h=\Phi(1)$, where $h$ is the class number, $R$ is the regulator and $C$ is a constant. If $\Delta<0, C=2 \pi / \sqrt{|\Delta|}$ and $R=\log \epsilon_{1}$, where $\epsilon_{1}(>1)$ is the fundamental unit of $K$.

Received July 28, 1975; revised October 7, 1975.

AMS (MOS) subject classifications (1970). Primary 12A50, 12 A30; Secondary 12-04, 12 A70. 
If $D$ is an integer which is not a perfect cube and $K=Q\left(D^{1 / 3}\right)$, the cubic field formed by adjoining $D^{1 / 3}$ to the rationals, we call $K$ a pure cubic field. Let $D$ be a cube free integer and let $D=a b^{2}$, where $a, b$ are square free. We have $\Delta=-3 k^{2}$, where

$$
k=\left\{\begin{array}{lll}
3 a b & \text { when } a^{2} \not \equiv b^{2} & (\bmod 9) \\
a b & \text { when } a^{2} \equiv b^{2} & (\bmod 9)
\end{array}\right.
$$

If $a^{2} \not \equiv b^{2}(\bmod 9)$, we say (after Dedekind) that $K$ is of type 1 ; otherwise, we say that $K$ is of type 2 .

In $K, \alpha(j)$ is a multiplicative function with $\alpha(1)=1$;

$$
\begin{aligned}
& \alpha\left(3^{n}\right)= \begin{cases}0 & \text { for } K \text { of type } 1, \\
1 & \text { for } K \text { of type } 2 ;\end{cases} \\
& \alpha\left(p^{n}\right)=0, \quad p \text { is a prime, } p \neq 3, \text { and } p \mid k .
\end{aligned}
$$

If $p$ is a prime and $p \equiv-1(\bmod 3), p \nmid k$,

$$
\alpha\left(p^{n}\right)= \begin{cases}1, & n \text { even } \\ 0, & n \text { odd }\end{cases}
$$

If $p$ is a prime and $p \equiv 1(\bmod 3), p \nmid k$,

$$
\alpha\left(p^{n}\right)=1+n, \quad \text { when }(D \mid p)_{3}=1
$$

otherwise,

$$
\alpha\left(p^{n}\right)=\left\{\begin{array}{rll}
1, & n \equiv 0 & (\bmod 3) \\
-1, & n \equiv 1 & (\bmod 3) \\
0, & n \equiv-1 & (\bmod 3)
\end{array}\right.
$$

Mention should also be made of a special divisibility property of $h$. In order to do this we first need some notation.

Let the number of distinct primes which divide $k$ be $\omega$, the number of distinct primes of the form $3 t+1$ which divide $k$ be $\omega_{1}$, and the number of distinct primes dividing $k$ which are congruent to either \pm 2 or \pm 4 modulo 9 be $\omega_{0}$. If $\omega_{0}=0$, put $\epsilon=0$; otherwise, put $\epsilon=1$; also, put $\omega^{*}=\omega-1-\epsilon$. Let $r_{n}$ be the $3^{n}$ rank of the class group of $K$, and let $r=\Sigma r_{n}$; then $3^{r} \| h$.

It has been shown [3], [10], [11], [8] that

$$
\max \left(\omega_{1}, \omega^{*}\right) \leqslant r_{1} \leqslant \omega_{1}+\omega^{*}
$$

Hence, if $\nu=\max \left(\omega_{1}, \omega^{*}\right)$, then $3^{\nu} \mid h$.

3. Estimation of $\Phi(1)$ for $Q\left(D^{1 / 3}\right)$. We start by defining the multiplicative functions $\beta(j)$ and $\beta^{*}(j)$. We first put $\beta^{*}(1)=\beta(1)=1$. If $p$ is a prime and $p \equiv-1(\bmod 3)$, we define

$$
\beta^{*}\left(p^{n}\right)=\beta\left(p^{n}\right)= \begin{cases}1, & n \text { even } \\ 0, & n \text { odd }\end{cases}
$$


If $p$ is a prime and $p \equiv 1(\bmod 3)$, we define

$$
\beta^{*}\left(p^{n}\right)=\beta\left(p^{n}\right)=1+n .
$$

Finally, we define $\beta^{*}\left(3^{n}\right)=0$ and $\beta\left(3^{n}\right)=1$. If $K$ is of type 1 , we have $-1 / 2 \beta^{*}(j) \leqslant$ $\alpha(j) \leqslant \beta^{*}(j)$; and if $K$ is of type 2 , we have $-1 / 2 \beta(j) \leqslant \alpha(j) \leqslant \beta(j)$.

We also define $M(m)=\max ((\beta(t) / t), t \geqslant m)$. It can be shown that

$$
M(m)=\max ((\beta(t) / t), \quad t=m, m+1, m+2, \ldots, 3 m) .
$$

This permits us to calculate $M(m)$ easily. It is evident that $|\alpha(j) / j| \leqslant M(m)$ for $j \geqslant m$.

Now $\Phi(s)$, the so-called Artin $L$ function, is an entire function (Dedekind) and satisfies the functional equation

$$
\Phi(1-s)=C^{-2 s+1}(\Gamma(s) / \Gamma(1-s)) \Phi(s) ;
$$

and from a result of Barrucand [2], it follows that

$$
\Phi(1)=\sum_{j=1}^{\infty} \frac{\alpha(j)}{j} e^{-j C}+C \sum_{j=1}^{\infty} \alpha(j) E(j C),
$$

where $E(y)=\int_{y}^{\infty}\left(e^{-x} / x\right) d x$. Putting

$$
A(n)=\sum_{j=1}^{n} \frac{\alpha(j)}{j} e^{-j C}+C \sum_{j=1}^{n} \alpha(j) E(j C),
$$

we have

$$
|\Phi(1)-A(n)| \leqslant S_{1}+C S_{2}+T_{1}+C T_{2},
$$

where

$$
\begin{array}{ll}
S_{1}=\sum_{j=n+1}^{m-1}\left|\frac{\alpha(j)}{j}\right| e^{-j C}, & S_{2}=\sum_{j=n+1}^{m-1}|\alpha(j)| E(j C), \\
T_{1}=\sum_{j=m}^{\infty}\left|\frac{\alpha(j)}{j}\right| e^{-j C}, & T_{2}=\sum_{j=m}^{\infty}|\alpha(j)| E(j C) .
\end{array}
$$

Since $e^{-y} / y>E(y)$ for $y>0$, it follows that

$$
T_{1}+C T_{2}<2 M(m) \sum_{j=m}^{\infty} e^{-j C}=\frac{2 M(m) e^{-(m-1) C}}{e^{C}-1}
$$

If we put

$$
\begin{aligned}
H(m) & =\frac{2 M(m)}{C} e^{-(m-1) C} /\left(e^{C}-1\right), \\
G(n, m, x) & =\frac{\sqrt{3} x}{2 \pi} \sum_{j=n+1}^{m-1} \beta(j) e^{-(2 \pi j / \sqrt{3} x)}+\sum_{j=n+1}^{m-1} \beta(j) E(2 \pi j / \sqrt{3} x), \\
G^{*}(n, m, x) & =\frac{3 \sqrt{3} x}{2 \pi} \sum_{j=n+1}^{m-1} \beta^{*}(j) e^{-(2 \pi j / 3 \sqrt{3} x)}+\sum_{j=n+1}^{m-1} \beta^{*}(j) E(2 \pi j / 3 \sqrt{3} x),
\end{aligned}
$$


we see that

$$
\left|\frac{\Phi(1)}{C}-\frac{A(n)}{C}\right|< \begin{cases}G^{*}(n, m, x)+H(m) & \text { if } K \text { is of type } 1 \\ G(n, m, x)+H(m) & \text { if } K \text { is of type } 2\end{cases}
$$

If $n$ is selected such that

$$
|\Phi(1) / C-A(n) / C|<1 / 23^{\nu} R
$$

then $h$ is the unique integer in the interval $\left(h^{*}-1 / 23^{\nu}, h^{*}+1 / 23^{\nu}\right)$ such that $3^{\nu} \mid h$. Here $h^{*}=A(n) / R C$.

4. Tabulation of $h$ for $D=2,3, \ldots, 9999$. Values of $M(m)$ were calculated for $m=1,2, \ldots, 10^{5}$ and it was seen that for $D<10^{4}$ and $m=\min \left([25 / C], 10^{5}\right)$, the value of $H(m)$ was usually less than .1. The functions $G(n, m, x)$ and $G^{*}(n, m, x)$ were also tabulated for $x=500(500) 10^{4}$ and $n=x / 10,2 x / 10,3 x / 10, \ldots, 2 x$ by putting

$$
m=\min \left(\left[\frac{25 \sqrt{3} x}{2 \pi}\right], 10^{5}\right) \text { for } G
$$

and

$$
m=\min \left(\left[\frac{75 \sqrt{3 x}}{2 \pi}\right], 10^{5}\right) \text { for } G^{*}
$$

All of these tables were calculated by computer and then stored on a disk file for easy access. The following computer algorithm was then used to determine $h$.

For any value of $D$, the computer first calculated the value of $R$ by using Voronoi's Algorithm (see [4]). It then put $x$ equal to the first multiple of 500 which exceeded $a b$ and selected $n$ from the stored tables of $G, G^{*}$ and $M$ to be the least value of $i x / 10$ $(i=1,2,3, \ldots, 20)$ such that

$$
1 / 23^{\nu} R> \begin{cases}G^{*}(n, m, x)+H(m)+.05 & \text { when } K \text { is of type } 1 \\ G(n, m, x)+H(m)+.05 & \text { when } K \text { is of type } 2\end{cases}
$$

With this value of $n, A(n)$ and subsequently $h$ were easily computed.

The algorithm was implemented on an IBM/370-158 computer and run for all values of $D$ such that $a>b$ between 1 and 10,000. The programs were written in FORTRAN with special assembler language routines being used for evaluating $E(j C)$ and $\alpha(j)$; also, all calculations were performed in double precision. The calculation of all the regulators required eight hours of CPU time and four additional hours were required for the evaluation of the class numbers.

Some indication of the speed of this method is provided by noting that the technique described in [4] required 8 minutes to evaluate the regulators for $Q\left(D^{1 / 3}\right)$ for $D$ $=2,3, \ldots, 999$ and 42 additional minutes to evaluate the class numbers. Our current method required 8 minutes to obtain the regulators and only 5 additional minutes to obtain the class numbers for these values of $D$. 
5. Evaluation of $h$ by Using the Euler Product. Another formula for $\zeta_{K}(1) / \zeta(1)$, where $K=Q\left(D^{1 / 3}\right)$, is given by the Euler product

$$
\Phi(1)=\zeta_{K}(1) / \zeta(1)=\prod_{q} f(q)
$$

where the product is taken over all the primes. Thus, for a given value of $D$, we define $f(q)$ for prime values of $q$ by the following formulas:

$$
\begin{aligned}
& \begin{array}{r}
\text { if } q \mid k, \quad f(q)=1 ; \\
\text { if } 3 \nmid k, \quad f(3)=3 / 2 ;
\end{array} \\
\text { if } q \equiv-1(\bmod 3), & f(q)=q^{2} /\left(q^{2}-1\right) ; \\
\text { if } q \equiv 1(\bmod 3), & f(q)= \begin{cases}q^{2} /(q-1)^{2} & \text { when }(D \mid q)_{3}=1, \\
q^{2} /\left(q^{2}+q+1\right) & \text { when }(D \mid q)_{3} \neq 1 .\end{cases}
\end{aligned}
$$

If we put the partial product $P(Q)=\Pi_{q}^{Q} f(q)$, we can estimate a value for $\Phi(1)$ by using a sufficiently large value of $Q$. The difficulty in using this formula lies in determining how big $Q$ should be. In [15] Shanks made use of a similar Euler product to obtain class numbers for some special cubic fields. He (private communication) evaluated his partial Euler products using the first 500,1000, 1500, etc., primes. When his estimate for the class number remained within .1 of the same integer for 6 consecutive partial Euler products, he took this integer as the class number.

We used this same criterion for estimating $\Phi(1)$ and discovered that in most cases only 3000 primes were needed in order to evaluate $h$. This method is very simple to program and executes from two to five times more rapidly than the technique described in Sections 3 and 4. It must, however, be emphasized that this procedure is not as rigorous as our first method. We cannot obtain bounds for the error on using the partial product that are as useful as the bounds we can obtain using our formulas for $A(n)$. The criterion which we do use for estimating $\Phi(1)$ by $P(Q)$ is one which is simply convenient for our calculations.

If, after Neild and Shanks [13], we put $E(Q)=1000(P(Q)-\Phi(1)) / \Phi(1)$, the relative error in parts per thousand on using $P(Q)$ to approximate $\Phi(1)$; and if we use 5000 primes to evaluate $E(Q)$, we get the following distribution for $E(Q)$ for the 166 pure cubic fields $Q\left(D^{1 / 3}\right)$ where $8000 \leqslant D \leqslant 8200$.

$$
Q=48611
$$

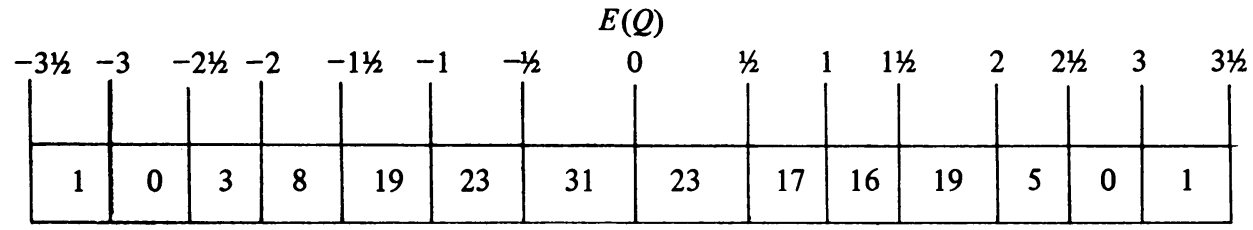

This distribution is typical of the sort of distribution we get for $E(Q)$ for the pure cubic fields $Q\left(D^{1 / 3}\right)\left(2 \leqslant D \leqslant 10^{4}\right)$.

6. Some Results of the Calculations. A large table, giving for each $D$ between 1 and $10^{4}$ such that $a>b$, the value of $k$, the length of Voronoi's continued fraction 
period for $D^{1 / 3}$, the regulator of $Q\left(D^{1 / 3}\right)$, the class number, and $\Phi(1)$, has been deposited in the U.M.T. file. We present here some selected results from that table. In Table 1 we give each value of $h$ which occurs in the large table, the frequency $g(h)$ with which this $h$ occurs, and the least value of $D$ such that $h$ is the class number for $Q\left(D^{1 / 3}\right)$.

If in this table we had presented, instead of $D$, the least value of $|\Delta|$ (in the range of $\Delta$ being considered) such that $h$ is the class number of the pure cubic field with discriminant $\Delta$, we would find that in most cases this value of $\Delta$ would correspond to the discriminant of the cubic field $Q\left(D^{1 / 3}\right)$ for the already given value of $D$. There are, however, several exceptions to this; for example, if $h=4$, the least $D$ value is 113 , but the least $|\Delta|$ is $3 \cdot 233^{2}$ not $3 \cdot 339^{2}$.

TABLE 1

\begin{tabular}{|c|c|c|c|c|c|}
\hline$h$ & $g(h)$ & $D$ & $h$ & $g(h)$ & $D$ \\
\hline 1 & 596 & 2 & 33 & 19 & 1618 \\
\hline 2 & 285 & 11 & 34 & 1 & 1719 \\
\hline 3 & 1847 & 7 & 36 & 262 & 322 \\
\hline 4 & 87 & 113 & 37 & 2 & 5545 \\
\hline 5 & 37 & 263 & 39 & 7 & 2597 \\
\hline 6 & 952 & 39 & 40 & 2 & 2733 \\
\hline 7 & 26 & 235 & 41 & 1 & 6659 \\
\hline 8 & 32 & 141 & 42 & 21 & 515 \\
\hline 9 & 1258 & 70 & 44 & 2 & 4817 \\
\hline 10 & 9 & 303 & 45 & 68 & 763 \\
\hline 11 & 7 & 2348 & 48 & 30 & 561 \\
\hline 12 & 359 & 43 & $\begin{array}{c}49 \\
51\end{array}$ & $\begin{array}{l}1 \\
4\end{array}$ & $\begin{array}{l}8171 \\
1037\end{array}$ \\
\hline 13 & 5 & 1049 & 52 & 1 & 4793 \\
\hline 14 & 7 & 514 & 54 & 172 & 614 \\
\hline 15 & 97 & 267 & 56 & 2 & 857 \\
\hline 16 & 9 & 681 & 57 & 5 & 1541 \\
\hline 17 & 1 & 8511 & 58 & 1 & 6814 \\
\hline 18 & 674 & 65 & 60 & 14 & 997 \\
\hline 19 & 2 & 667 & 63 & 29 & 1005 \\
\hline 20 & 6 & 761 & 64 & 1 & 9749 \\
\hline 21 & 51 & 213 & 66 & 5 & 3482 \\
\hline 22 & 4 & 281 & 68 & 1 & 9521 \\
\hline 24 & 96 & 229 & 69 & 4 & 3590 \\
\hline 26 & 1 & 3403 & 70 & 1 & 3467 \\
\hline 27 & 385 & 182 & 71 & 1 & 3539 \\
\hline 28 & 6 & 509 & 72 & 90 & 741 \\
\hline 30 & 38 & 524 & 74 & 1 & 3581 \\
\hline 32 & 3 & 2399 & 75 & 3 & 1657 \\
\hline
\end{tabular}


TABLE 1 (continued)

\begin{tabular}{|c|c|c|c|c|c|}
\hline$h$ & $g(h)$ & $D$ & $h$ & $g(h)$ & $D$ \\
\hline 78 & 9 & 1801 & 216 & 17 & 2765 \\
\hline 80 & 1 & 4799 & 222 & 1 & 5823 \\
\hline 81 & 77 & 1298 & 225 & 3 & 5362 \\
\hline 84 & 9 & 1737 & 230 & 1 & 4451 \\
\hline 87 & 2 & 4103 & 240 & 2 & 5835 \\
\hline 90 & 27 & 970 & 243 & 6 & 3913 \\
\hline 93 & 1 & 2748 & 252 & 3 & 2786 \\
\hline 96 & 5 & 4307 & 254 & 1 & 8002 \\
\hline 99 & 8 & 995 & 255 & 1 & 2751 \\
\hline 102 & 4 & 2374 & 264 & 1 & 7297 \\
\hline 105 & 4 & 2737 & 270 & 4 & 4593 \\
\hline 108 & 87 & 511 & 276 & 1 & 4093 \\
\hline 111 & 2 & 5737 & 279 & 1 & 5149 \\
\hline 117 & 4 & 5215 & 288 & 1 & 5826 \\
\hline 120 & 10 & 1727 & 297 & 3 & 6487 \\
\hline 126 & 23 & 1141 & 300 & 1 & 9931 \\
\hline 127 & 1 & 2741 & 306 & 2 & 4694 \\
\hline 128 & 1 & 5987 & 312 & 1 & 9938 \\
\hline 129 & 1 & 2946 & 315 & 2 & 5359 \\
\hline 132 & 3 & 3045 & 324 & 10 & 2198 \\
\hline 135 & 11 & 1015 & 336 & 1 & 8005 \\
\hline 136 & 1 & 3209 & 342 & 1 & 3907 \\
\hline 141 & 1 & 6991 & 351 & 3 & 3605 \\
\hline 144 & 17 & 1730 & 360 & 1 & 7985 \\
\hline 150 & 1 & 8431 & 369 & 1 & 5829 \\
\hline 153 & 2 & 3661 & 372 & 1 & 7133 \\
\hline 154 & 1 & 9041 & 378 & 3 & 3155 \\
\hline 156 & 2 & 7461 & 390 & 1 & 9591 \\
\hline 162 & 36 & 813 & 396 & 2 & 7997 \\
\hline 168 & 2 & 2747 & 405 & 6 & 7970 \\
\hline 171 & 1 & 9198 & 432 & 4 & 6878 \\
\hline 175 & 1 & 5711 & 435 & 1 & 8006 \\
\hline 180 & 12 & 2702 & 459 & 1 & 9254 \\
\hline 186 & 1 & 4099 & 480 & 1 & 7415 \\
\hline 189 & 7 & 6430 & 486 & 4 & 6162 \\
\hline 192 & 2 & 7925 & 576 & 1 & 4291 \\
\hline 198 & 7 & 3374 & 585 & 1 & 9262 \\
\hline 201 & 2 & 2723 & 612 & 1 & 7995 \\
\hline
\end{tabular}


TABLE 1 (continued)

\begin{tabular}{lllrrl}
\hline$h$ & $g(h)$ & $D$ & $h$ & $g(h)$ & \multicolumn{1}{c}{$D$} \\
\hline 630 & 1 & 9933 & 972 & 1 & 9709 \\
648 & 1 & 4097 & 1017 & 1 & 8615 \\
696 & 1 & 5503 & 1170 & 1 & 7999 \\
747 & 1 & 2743 & 1296 & 1 & 8827 \\
756 & 1 & 8030 & & &
\end{tabular}

In Table $1 \mathrm{~A}$ we give the frequency $v(p), p \leqslant 19$, of the cases where $p \mid h$ and the percentage of the occurrences.

TABLE 1A

\begin{tabular}{|c|c|c||c|c|c|}
\hline$p$ & $v(p)$ & $\%$ & $p$ & $v(p)$ & $\%$ \\
\hline 2 & 3510 & 43.22 & 11 & 62 & 0.76 \\
3 & 6954 & 85.62 & 13 & 36 & 0.44 \\
5 & 369 & 4.54 & 17 & 19 & 0.23 \\
7 & 202 & 2.49 & 19 & 9 & 0.11 \\
\hline
\end{tabular}

Moreover, we have 7409 fields such that $h=2^{\alpha} 3^{\beta}$ and 713 fields such that some prime $>3$ divides $h$.

In Table 2 we give the number $m$ of values of $D$ in the ranges $1000(i-1)$ to $1000 i(i=1,2,3, \ldots, 10)$ for which the class number of $Q\left(D^{1 / 3}\right)$ is unity.

TABLE 2

\begin{tabular}{|rl|ll|}
\hline Range of $D$ & $m$ & Range of $D$ & $m$ \\
\hline $0-1000$ & 98 & $5000-6000$ & 55 \\
$1000-2000$ & 64 & $6000-7000$ & 49 \\
$2000-3000$ & 56 & $7000-8000$ & 54 \\
$3000-4000$ & 61 & $8000-9000$ & 44 \\
$4000-5000$ & 65 & $9000-10000$ & 50 \\
\hline
\end{tabular}

Denote by $R(d)$ and $\Phi_{d}(1)$ the value of the regulator and the value of $\Phi(1)$ for $Q\left(d^{1 / 3}\right)$, respectively. In Table 3 we give those values of $D$ and $\Phi_{D}(1)$ such that

$$
\Phi_{D}(1)<\Phi_{d}(1) \text { for all } 0<d<D \text {. }
$$

TABLE 3

\begin{tabular}{|rc|cc|}
\hline$D$ & $\Phi(1)$ & $D$ & $\Phi(1)$ \\
\hline 2 & 0.8146240593 & 1510 & 0.6672743355 \\
74 & 0.7323553491 & 2740 & 0.6496367445 \\
166 & 0.6767319520 & 4630 & 0.6251252454 \\
276 & 0.6733957020 & 9770 & 0.6199747135 \\
830 & 0.6684533198 & & \\
\hline
\end{tabular}


In Table 4 we give those values of $D$ and $\Phi_{D}(1)$ such that $\Phi_{D}(1)>\Phi_{d}(1)$ for all $0<d<D$.

\begin{tabular}{rcrc}
\multicolumn{4}{c}{ TABLE 4 } \\
\hline$D$ & $\Phi(1)$ & $D$ & $\Phi(1)$ \\
\hline 2 & 0.8146240593 & 307 & 2.8227637445 \\
3 & 1.0176145615 & 559 & 2.9367139608 \\
5 & 1.1637304168 & 629 & 2.9626689819 \\
6 & 1.1668639154 & 827 & 2.9707482692 \\
7 & 1.2650247640 & 883 & 3.0623905474 \\
13 & 1.5743940270 & 1009 & 3.0683245965 \\
29 & 1.6791537873 & 1457 & 3.0931407438 \\
35 & 1.7499243062 & 1513 & 3.3383074285 \\
53 & 1.8171807443 & 1945 & 3.3680857678 \\
55 & 2.1254129939 & 3457 & 3.5990411752 \\
71 & 2.2034566301 & 4789 & 3.6257791705 \\
127 & 2.3311172521 & 5669 & 3.7254983552 \\
181 & 2.6622437425 & 9017 & 3.8119134914 \\
\hline
\end{tabular}

Finally, in Table 5 we give those values of $D, R(D)$ and $J$, the length of Voronoi's algorithm period of $D^{1 / 3}$, such that

$$
R(D)>R(d) \text { for all } 0<d<D
$$

TABLE 5

\begin{tabular}{rrrrrr}
\hline \multicolumn{1}{c}{$D$} & \multicolumn{1}{c}{$R(D)$} & $J$ & \multicolumn{1}{c}{$D$} & \multicolumn{1}{c}{$R(D)$} & \multicolumn{1}{c}{$J$} \\
\hline 2 & 1.347377348 & 1 & 951 & 1521.5849715 & 1352 \\
3 & 2.524681405 & 3 & 1163 & 1818.3574652 & 1595 \\
5 & 4.811986540 & 5 & 1301 & 2549.9434350 & 2307 \\
6 & 5.789932142 & 5 & 1721 & 3669.3791260 & 3320 \\
15 & 9.692951678 & 5 & 2003 & 3675.2829265 & 3255 \\
23 & 22.595071214 & 21 & 2283 & 4340.6136141 & 3959 \\
29 & 40.270821121 & 35 & 2927 & 4671.7189737 & 4076 \\
41 & 56.289370200 & 49 & 3543 & 4681.9661909 & 4096 \\
69 & 103.810793808 & 100 & 3557 & 6170.2103314 & 5393 \\
137 & 134.626355970 & 122 & 3821 & 7106.2863230 & 6388 \\
167 & 220.571825346 & 206 & 3921 & 8909.1586123 & 8014 \\
227 & 224.944023983 & 206 & 4523 & 9440.9625040 & 8545 \\
239 & 431.942240996 & 390 & 5153 & 9766.3676264 & 8576 \\
411 & 555.643020852 & 488 & 5433 & 12019.3087665 & 10702 \\
419 & 711.993772506 & 646 & 6999 & 13777.0095919 & 12338 \\
447 & 778.588027713 & 719 & 8093 & 15231.6425197 & 13591 \\
573 & 991.930184538 & 877 & 8429 & 17248.5337519 & 15481 \\
771 & 1321.452703846 & 1202 & & & \\
\hline
\end{tabular}


7. Some Results on the Class Structure. We shall employ in this section the same notation as that introduced in Section 2. We also define $j=e^{2 \pi i / 3}$ and let the symbols $p, q$ represent primes. By $(n \mid l)_{3}$ we represent a cubic character modulo $l$, where $l$ is either a prime of the form $1+3 t$ or $l=9$. Since $(n \mid l)_{3}=j, j^{2}$, or 1 , the character is completely determined when we select a cubic nonresidue $n_{0}$ and define the value of $\left(n_{0} \mid l\right)$ as either $j$ or $j^{2}$.

From the inequality (2.1), we see that $r_{1}$ is known exactly in four cases.

(1) $\omega_{1}=0, r_{1}=\omega^{*}$.

(2) $D=p, \quad p \equiv 1(\bmod 9), \quad r_{1}=1$.

(3) $D=p, 3 p, 9 p, \quad p \equiv 4,7(\bmod 9), \quad r_{1}=1$.

(4) $D=p p^{\prime}, p p^{\prime 2}, \quad D \equiv 1(\bmod 9), \quad \epsilon=1, \quad r_{1}=2$.

In the other cases, it was shown by Gerth [8] and Kobayashi [11] that $r_{1}=\omega_{1}+\omega^{*}$ - $s$, where $s$ can be computed by evaluating Hilbert symbols in $Q(\sqrt{-3})$ or, what is the same thing, cubic residuacity symbols in $Q(\sqrt{-3})$. The case of $\epsilon=0$ is troublesome; consequently, we shall restrict ourselves to the case of $\epsilon=1$ only. If $\omega_{1}=1$, we have $k=p k^{*}$, where $p$ is a prime congruent to 1 modulo 3 and no prime which divides $k^{*}$ is congruent to 1 modulo 3 . From this we can deduce

THEOREM 1. If $D=p, 3 p, 9 p$, where $p \equiv 4,7(\bmod 9)$ and $(3 \mid p)_{3} \neq 1$, then $r_{1}$ $=1, r_{2}=0$ and, consequently, $3 \| h$.

THEOREM 2. If $D=p q$, where $p \equiv-q \equiv 1(\bmod 3),(q \mid p)_{3} \neq 1$, and $\epsilon=1$, then $r_{1}=1, r_{2}=0$.

THEOREM 3. If $D=p q \not \equiv \pm 1(\bmod 9)$, where $q \equiv 2(\bmod 3), p \equiv 4,7(\bmod 9)$, $(3 \mid p)_{3}=1$ and $(q \mid p)_{3} \neq 1$, the ideal ramifying (3) in $K=Q\left(D^{1 / 3}\right)$ is principal.

To prove Theorem 1, we use the fact that $r_{1}=1$ and remark that we have a rational genus character system, that is if $p$ is a principal ideal in $K=Q\left(D^{1 / 3}\right)$, then $(N(p) \mid p)_{3}=1$. Since $(3 \mid p)_{3} \neq 1$, we have an "ambiguous" class $\mathrm{C} 1$ such that if $p^{*}$ $\in \mathrm{C} 1$, then $\left(N\left(p^{*}\right) \mid p\right)_{3}=j-\mathrm{a}$ contradiction. (The proof may be compared to the wellknown proof of the result that $2 \| h(\sqrt{-p})$ when $p \equiv 5(\bmod 8)$.)

TABLE 6

\begin{tabular}{|cccccc|}
\hline$p, q$ & $(p \mid 9)_{3}$ & $(q \mid 9)_{3}$ & $(3 \mid p)_{3}(3 q \mid p)_{3}$ & $h$ \\
\hline 7,2 & $j$ & $j$ & $j$ & 1 & 3 \\
13,2 & $j$ & $j^{2}$ & $j$ & $j^{2}$ & 3 \\
7,11 & $j$ & $j$ & $j$ & $j$ & 3 \\
61,2 & $j$ & $j$ & 1 & $j$ & 12 \\
67,2 & $j$ & $j^{2}$ & 1 & $j$ & 3 \\
73,2 & 1 & $j$ & 1 & $j$ & 3 \\
19,2 & 1 & $j$ & $j$ & $j^{2}$ & 3 \\
19,23 & 1 & $j$ & $j$ & 1 & 6 \\
7,17 & $j$ & 1 & $j$ & $j^{2}$ & 3 \\
13,17 & $j$ & 1 & $j$ & 1 & 3 \\
61,17 & $j$ & 1 & 1 & $j$ & 51 \\
\hline
\end{tabular}


To prove Theorem 2, we require a list of $D$ values in which all possibilities for the distribution of characters are present. It can be verified in Table 6 that, if $(n \mid p)_{3}$ is defined by $(q \mid p)_{3}=j$, in each case we have $3 \| h$. The proof that $r_{2}=0$ is similar to that of Theorem 1. Theorem 3 is a simple corollary of Theorem 2.

8. Remarks and Conjectures. We present in this section some conjectures based on observations made of various phenomena in the table and verified for all $D \leqslant 9999$.

Conjecture 1. If $D=p, 3 p, 9 p$, where $p \equiv 4,7(\bmod 9)$ and $(3 \mid p)_{3}=1$, then $r_{2}=0$.

Conjecture 2. If $D=p p^{\prime}$, where $p \equiv p^{\prime} \equiv 1(\bmod 3)$ and $D \not \equiv 1(\bmod 9)$, then $27 \mid h$ if and only if $\left(p \mid p^{\prime}\right)_{3}=\left(p^{\prime} \mid p\right)_{3}$ or if $p \equiv 1(\bmod 9),(3 \mid p)_{3}=\left(p^{\prime} \mid p\right)_{3}=1$.

Both Theorem 1 and Conjecture 1 are false if $p \equiv 1(\bmod 9)$; for, in some cases we have $9 \mid h$ which implies that $r_{2} \geqslant 1$. This happens for $p=199,271, \ldots$ Similarly, Conjecture 2 becomes false when $p p^{\prime} \equiv 1(\bmod 9)$ even if $\epsilon=1$, as may be inferred from the fact that $h=27$ for $D=7.31$.

With the exception of the results of Theorems 1 and 2, almost nothing appears to be known about $r_{2}$; however, it is perhaps worth mentioning that $r_{2}=1$ for some $D=$ $q q^{\prime} q^{\prime \prime}$, where $q \equiv q^{\prime} \equiv q^{\prime \prime} \equiv-1(\bmod 3)$. For example if $D=2 \cdot 5 \cdot 101, h=54$ and $r_{1}$ $=2$.

Conjecture 3. If $p \equiv 1(\bmod 9)$ and $q \equiv-1(\bmod 9),(q \mid p)_{3} \neq 1, D=p q$, then $r_{1}=1$ and $r_{2}=0$.

Conjecture 4. If $q \equiv q^{\prime} \equiv 2$ or $5(\bmod 9)$ then $r_{1}=1, r_{2}=0$.

A part of these conjectures may be proved by using some unpublished results of Gerth [9], but no theory concerning $r_{2}$ seems to be known. For example, if $D=p \equiv$ $1(\bmod 9)$, we may find $r_{2}=0(p=19)$ or $r_{2}=1(p=199)$.

Generally, the class number remains small; but in some rare cases it may be unusually large as in the case of $D=8827, h=1296$.

9. Acknowledgment. The authors would like to thank Daniel Shanks for making several suggestions concerning this work and for pointing out some errors which occurred in our early computer runs.

151 Rue Chateau des Rentiers

Paris 75013, France

Department of Computer Science

University of Manitoba

Winnipeg, Manitoba, Canada R3T 2N2

1. I. O. ANGELL, “A table of complex cubic fields," Bull. London Math. Soc., v. 5, 1973, pp. 37-38. MR 47 \#6648.

2. PIERRE BARRUCAND, "Sur certaines séries de Dirichlet," C. R. Acad. Sci. Paris Sér. $A-B$, v. 269, 1969, pp. A294-A296. MR $40 \# 101$.

3. P. BARRUCAND \& H. COHN, "A rational genus, class number divisibility, and unit theory for pure cubic fields," J. Number Theory, v. 2, 1970, pp. 7-21. MR 40 \#2643.

4. B. D. BEACH, H. C. WILLIAMS \& C. R. ZARNKE, "Some computer results on units in quadratic and cubic fields," Proc. Twenty-Fifth Summer Meeting of the Canadian Math. Congress (Lakehead Univ., Thunder Bay, Ont., 1971), Lakehead Univ., Thunder Bay, Ont., 1971, pp. 609648. MR 49 \#2656. 
5. J. W. S. CASSELS, "The rational solutions of the diophantine equation $Y^{2}=X^{3}-D$," Acta Math., v. 82, 1950, pp. 243-273. MR 12, 11.

6. HARVEY COHN, “A numerical study of Dedekind's cubic class number formula," J. Res. Nat. Bur. Standards, v. 59, 1957, pp. 265-271. MR 19, 944.

7. R. DEDEKIND, “Über die Anzahl der Idealklassen in reinen kubischen Zahlkörpern," $J$. Reine Angew. Math., v. 121, 1900, pp. 40-123.

8. FRANK GERTH III, "Ranks of Sylow 3 subgroups of ideal class groups of certain cubic fields,” Bull. Amer. Math. Soc., v. 79, 1973, pp. 521-525.

9. FRANK GERTH III, "The class structure of the pure cubic field." (Unpublished.)

10. TAIRA HONDA, "Pure cubic fields whose class numbers are multiples of three," J. Number Theory, v. 3, 1971 , pp. 7-12. MR 45 \#1877.

11. S. KOBAYASHI, "On the 3-rank of the ideal class groups of certain pure cubic fields," J. Fac. Sci. Univ. Tokyo Sect. I A Math., v. 20, 1973, pp. 209-216. MR 48 \#3919.

12. A. MARKOV, "Sur les nombres entiers dépendants d'une racine cubique d'un nombre entier ordinaire," Mem. Acad. Imp. Sci. St. Petersburg, v. (7) 38, 1892, no. 9, pp. 1-37.

13. CAROL NEILD \& DANIEL SHANKS, "On the 3-rank of quadratic fields and the Euler product," Math. Comp., v. 28, 1974, pp. 279-291.

14. ERNST S. SELMER, "Tables for the purely cubic field $K(\sqrt[3]{m})$," Avh. Norske Vid. Akad. Oslo I, v. 1955, no. 5. MR 18, 286.

15. DANIEL SHANKS, "The simplest cubic fields," Math. Comp., v. 28, 1974, pp. $1137-$ 1152. 